\title{
PATRIMONIALISATION DES MÉMOIRES DOULOUREUSES : ANCRAGES ET MOBILITÉS, RACINES ET RHIZOMES
}

Dominique Chevalier

Presses de Sciences Po (P.F.N.S.P.) | « Autrepart »

2016/2 N 78-79 | pages 235 à 255

ISSN 1278-3986

ISBN 9782724634402

Article disponible en ligne à l'adresse :

https://www.cairn.info/revue-autrepart-2016-2-page-235.htm

\section{Pour citer cet article :}

Dominique Chevalier, «Patrimonialisation des mémoires douloureuses : ancrages et mobilités, racines et rhizomes », Autrepart 2016/2 (N 78-79), p. 235-255.

DOI 10.3917/autr.078.0235

Distribution électronique Cairn.info pour Presses de Sciences Po (P.F.N.S.P.).

(C) Presses de Sciences Po (P.F.N.S.P.). Tous droits réservés pour tous pays.

La reproduction ou représentation de cet article, notamment par photocopie, n'est autorisée que dans les limites des conditions générales d'utilisation du site ou, le cas échéant, des conditions générales de la licence souscrite par votre établissement. Toute autre reproduction ou représentation, en tout ou partie, sous quelque forme et de quelque manière que ce soit, est interdite sauf accord préalable et écrit de l'éditeur, en dehors des cas prévus par la législation en vigueur en France. Il est précisé que son stockage dans une base de données est également interdit. 


\title{
Patrimonialisation des mémoires douloureuses : ancrages et mobilités, racines et rhizomes
}

\author{
Dominique Chevalier* \\ «Le passé n'est jamais mort. Il n'est même jamais passé. » \\ William Faulkner
}

Étymologiquement, le terme "patrimoine » suggère l'idée d'un héritage, légué par les générations précédentes, à transmettre intact aux générations futures. «On ne peut donner que deux choses à ses enfants : des racines et des ailes », assure un proverbe juif: des racines pour puiser la substance vitale qui fonde chaque individu et compose son identité et sa mémoire et des ailes pour s'élever, s'envoler vers son propre destin avec légèreté et spiritualité.

La racine pater du terme patrimoine évoque explicitement cette transmission par filiation. En raison du génocide et des crimes de masse perpétrés pendant la Seconde Guerre mondiale, six millions de racines et d'innombrables ramifications et filaments ont été détruits et annihilés. Maillons d'une chaine qui unit les vivants et les morts, les musées et mémoriaux consacrés aux mémoires douloureuses de la Shoah apparaissent de ce point de vue à la fois comme une manifestation de l'importance du Zakhor [Yerushalmi, 1984], régulièrement rappelé et invoqué dans la Bible hébraïque, et comme une réponse spatiale, patrimoniale, mémorielle et géopolitique de nombreux acteurs de la scène politique et de la société civile. Les musées consacrés aux mémoires douloureuses répondent en effet à une utilité sociale forte : ils pansent ${ }^{1}$ et font penser.

Avec l'émergence, à la suite du procès Barbie (1987), d'une frénésie commémorative et du devoir de mémoire [Lalieu, 2001 ; Ledoux, 2012], le passé, ce «pays étranger » pour reprendre le titre de David Lowenthal [1985], est devenu une force plus puissante que jamais pour envisager le monde contemporain et à venir ; ce dernier montre en effet combien l'évocation désormais récurrente du passé dans l'espace public occasionne un changement de signification dans

* MCF, HDR, université Claude Bernard-ESPÉ Lyon 1.

1. Voir à ce sujet les travaux sur la résilience [Cyrulnik, 2001] et [Cyrulnik, Seron, 2004]. 
l'appréhension du patrimoine, lequel ne consiste plus seulement en une préservation des objets, mais devient une création, une construction en soi. Dès lors, le patrimoine n'est plus appréhendé comme un ensemble d'éléments ou d'artefacts transmis du passé au présent, mais comme un processus, une création permanente visant à répondre à des besoins contemporains [Ashworth, 2012, p. 187]. Ce passé, associé aux différentes formes de patrimoines qu'il embrasse et génère, présente des ressources à la fois culturelles, touristiques et mémorielles. Pour reprendre les propos d'Olivier Lazzarotti, « le tourisme dit culturel et sa rencontre avec le patrimoine participent à la construction du Monde contemporain » [Lazzarotti, 2010].

La question des mobilités apparaît centrale pour comprendre les diverses formes de patrimonialisation du passé des mémoires douloureuses de la Shoah. Les patrimonialisations sont plurielles, à la fois construites autour de mobilités temporelles pour retracer chronologiquement les mondes perdus d'avant la destruction des Juifs d'Europe, puis les ténèbres des centres de mise à mort, et finalement les reconstructions et itinéraires post-génocides; elles sont également articulées et produites par des mobilités géographiques idéelles ou matérielles de personnes, d'objets et de pratiques pour évoquer les nouveaux réseaux diasporiques, en Israël, en Amérique du Nord et du Sud, en Afrique du Sud ou en Nouvelle-Zélande après la Seconde Guerre mondiale. Enfin, les mobilités sont également mouvances, dans le sens où les mouvements, les turbulences, les circulations de personnes, d'objets et de mémoires plurielles contribuent finalement à façonner et à «labelliser » la mise en patrimoine d'autres types de mémoires douloureuses.

Ce boom mémoriel, dont certain(es) chercheur(es) déplorent l'omniprésence [Todorov, 1995] ou en critiquent «l'overextension » [Berliner, 2005] a infusé toutes les disciplines. La géographie, jusque-là assez peu concernée par le paradigme mémoriel, n'échappe pas à cet engouement; les politiques mémorielles et les mémoires politiques et sociales nourrissent et transforment en effet formidablement les espaces dans lesquels elles s'insèrent. Les différentes formes d'inscriptions patrimoniales et mémorielles de mémoires traumatiques plurielles constitueront le fil rouge de notre approche $^{2}$. Dans un premier temps, nous appréhenderons les mises en patrimoine des mémoires de la Shoah en tant que «label» pour d'autres mémoires [Chevalier, 2014a] et nous essaierons d'en comprendre les ressorts. Puis nous nous intéresserons plus particulièrement aux «entrepreneurs de mémoire » [Pollak, 1993 ; Gensburger, 2010] tels qu'architectes, artistes et touristes en les appréhendant en tant qu'agents, voire, agitateurs mémoriels. Enfin, nous interrogerons les liens existant entre massification touristique et marchandisation patrimoniale.

2. D'un point de vue méthodologique, j'ai systématiquement privilégié l'observation flottante [Pétonnet, 1982], beaucoup moins intrusive que des entretiens fussent-ils non directifs. En effet, comme j'ai rapidement pu le constater, les visiteurs des musées des mémoires douloureuses gardent entre eux une certaine distance, respectent les proxémies intimes et personnelles [Hall, 1978] de chacun(e) et limitent au maximum les interactions verbales. Mes entrées sur le terrain n'ont donc finalement pas été très compliquées. «Touriste » ordinaire et visiteuse anonyme parmi d'autres touristes et visiteur.es anonymes et ordinaires, j'ai ainsi pu fréquenter, souvent à plusieurs reprises, chaque musée des différents corpus et observé à loisir les dispositifs muséographiques et les réactions de mes semblables. 


\section{Mémoires de la Shoah et patrimonialisation : un « label » pour les autres mémoires douloureuses?}

Sur le plan matériel, oral et immatériel, la culture et le patrimoine liés à la Shoah constituent un exemple à la fois archétypal et douloureux de l'ensemble des caractéristiques définies par l'Unesco en $1997^{3}$. Aux innombrables documents d'archives, à la multitude d'objets et artefacts, aux témoignages écrits, oraux et vidéos de nombreux rescapés, il convient d'ajouter les importantes œuvres d'art aux fonctions à la fois artistiques, historiques, morales et psychologiques présentes dans les musées et dans les jardins qui les jouxtent parfois. L'architecture des édifices nationaux, construits ex nihilo hors des sites de déportation (et donc sans liens géographique et historique avec la Shoah), insérés au cœur de quelques grandes métropoles occidentales, doit relever un défi supplémentaire, celui de faire comprendre aux visiteurs, à travers la puissance évocatrice de leur dessin, la barbarie du dessein des nazis et de leurs complices. Bien que situé à des milliers de kilomètres des lieux de la Shoah, l'US Holocaust Memorial Museum de Washington a reçu plus de 40 millions de visiteurs depuis son ouverture en avril 1993. Aux fréquentations présentielles s'ajoutent les visites virtuelles : en 2015, le site Web du musée ${ }^{4}$ en a reçu plus de 25 millions, émanant de plus de 17 millions de visiteurs provenant de 239 pays. $43,6 \%$ de ces visites étaient des visites internationales.

Les ondes de choc de la Shoah [Lindenberg, Garapon, Padis, 2008] continuent de travailler les sociétés contemporaines. Ainsi que le remarque Serge Klarsfeld [2002, p. 13], «[les nazis] ont [...] marqué au plus profond de leur personnalité des hommes et des femmes qui sont nés bien après la Shoah ». Par sa complexité, par son envergure, son bilan et sa monstruosité la Shoah reste un défi pour la conscience morale de l'humanité. Comme le note Paul Ricœur [2000, p. 331-332], « il y aurait dans l'événement même quelque chose de si monstrueux qu'il mettrait en déroute tous les modes de représentation disponibles ». Diverses remémorations collectives tentent de penser cette béance dans l'histoire de l'humanité. Les commémorations sont plurielles [Perego, 2010], et les scénographies et rituels [Wahnich, 2007] mis en œuvre pour évoquer les souvenirs inspirent en partie, par leur efficience, les cérémonies commémoratives d'autres mémoires douloureuses.

Tout d'abord, sans doute faut-il voir des raisons juridiques à cette influence de la Shoah dans les commémorations d'autres mémoires douloureuses. En effet, en 1948, à la suite de la destruction des Juifs d'Europe, les notions de «crime contre l'humanité » et de " génocide » sont définies, dans la sphère du droit et de la justice pénale internationale, pour évoquer spécifiquement les formes de violences planifiées par les institutions politiques, en vue d'éradiquer une partie de la population en tant que telle. La Shoah apparaît clairement comme un paradigme de référence.

3. http://unesdoc.unesco.org/images/0010/001062/106215E.pdf (page consultée le 3 décembre 2016).

4. www.ushmm.org/ (page consultée le 3 décembre 2016). 
Dans le cas du génocide des Tutsis rwandais, dernière grande tuerie de masse du vingtième siècle, la nature génocidaire des crimes est évidente ; Hélène Dumas [2014] en a donné une grille de lecture claire, notamment en matière d'organisation administrative et d'investissement populaire à l'échelle de l'espace proche. En cent jours, entre le 7 avril et le 4 juillet 1994, huit cent mille à un million de Tutsis rwandais, hommes, femmes et enfants ont été tués dans ce pays qui comptait sept millions d'habitants. Telle la foudre, la haine s'est abattue sur eux au motif qu'ils étaient Tutsis. Jean-Pierre Chrétien et Claudine Vidal [2014] ont montré le caractère construit de l'opposition Hutus/Tutsis et le rôle du pouvoir colonial dans cette catégorisation. La plupart des victimes ont été massacrées durant les trois premières semaines, ce qui donne une idée de la radicalité de l'événement et des difficultés actuelles pour les rescapé(e)s de cohabiter à côté des bourreaux d'hier.

En 1995, plusieurs représentants de la mémoire de la Shoah, notamment Michaël Berenbaum, du Musée de l'Holocaust de Washington D.C. (USHMM) ou encore Efraim Zuroff du Centre Simon-Wiesenthal de Jérusalem, furent invités au Rwanda, en tant qu'experts, pour proposer des idées sur la gestion de la mémoire du génocide des Tutsis rwandais. D'ailleurs, à l'issue de la conférence internationale intitulée «Conférence internationale sur le Génocide. Impunité et Responsabilité », Efraim Zuroff devint conseiller du gouvernement rwandais dans la chasse aux génocidaires. L'année suivante, en 1996, c'est Paul Kagame, vice-président et ministre de la Défense, qui se déplace et effectue un séjour de quelques jours en Isräl. Au cours de ce voyage, il visite Yad Vashem et exprime le souhait de construire un Mémorial qui s'en inspirerait. Depuis lors, devenu membre d'honneur de la fondation Raoul Wallenberg, il a, à maintes occasions, affirmé la proximité de fait existant entre le Rwanda et Israël en raison du crime subi [Kaliski, 2004a]. Le mémorial du génocide, inauguré à Kigali en 2004, abrite les sépultures de quelque 250000 victimes. En voyage officiel en Afrique, le Premier ministre israélien s'est récemment rendu au Mémorial en compagnie de Paul Kagamé, devenu président de la République en 2000. Lors d'une conférence de presse, il a notamment déclaré : « Mon peuple connaît également la douleur d'un génocide. C'est un lien unique, même si c'est un lien qu'aucun de nos peuples ne souhaite avoir » [Perez, 2016].

Si cette «réelle affinité [entre les deux pays] pour des raisons historiques évidentes » [Clark, cité par Njanjo, 2016] s'explique à la fois par une affinité de souffrances et des considérations géopolitiques ${ }^{5}$, l'analogie ne se retrouve que partiellement dans les choix muséographiques. En effet, les mémoriaux rwandais exposent généralement, tout comme à Chœung Ek ou Tuol Sleng, principaux lieux mémoriaux du génocide perpétré par les Khmers rouges au Cambodge, de nombreux

5. Les relations entre Israël et le Rwanda se sont intensifiées depuis 2013. Les deux pays ont signé un accord de coopération en 2014 et en 2015, et le Rwanda a ouvert une ambassade à Tel-Aviv. Les liens entre les deux pays ne se bornent pas seulement à un passé marqué par le génocide. D'un point de vue plus pragmatique, Israël est perçu comme un partenaire intéressant, à un moment où les relations avec les États-Unis et le Royaume-Uni s'avèrent quelque peu tendues. Et par ailleurs, Israël cherche à s'assurer le soutien des pays africains, notamment dans les institutions internationales où l'État hébreu est critiqué pour son occupation des Territoires palestiniens ou ses activités nucléaires. 
restes humains. À quelques exceptions près, comme l'ossuaire de Douaumont ${ }^{6}$ par exemple, cette pratique de la monstration de restes humains demeure relativement étrangère aux usages occidentaux. Faut-il voir dans cette volonté d'exposer les ossements, une politique de mémoire traumatique qui cherche à maintenir vivace la présence de la violence du passé, comme le suggère Henri Rousso [2015] ?

Dans les musées et mémoriaux consacrés à la Shoah, les rites et les agencements mémoriels reposent certes sur des témoignages, des archives, l'exposition d'objets, etc., mais également sur la symbolisation et l'invocation des noms, soit sous la forme de «Mur des Noms » comme dans pratiquement chaque musée, soit sous la forme d'une longue litanie des noms comme dans le Mémorial des enfants de Yad Vashem ou le musée attenant au Mémorial aux Juifs assassinés d'Europe à Berlin notamment, ou encore lors de la cérémonie de Yom Ha Shoah.

Ces rituels commémorant la Destruction des Juifs d'Europe [Hilberg, 1988] permettent sans doute de saisir pourquoi et comment la Shoah est devenue un « modèle et un cadre référentiel » [Lapierre, 2007, p. 475] à part entière du « phénomène mémoriel » [Lavabre, 2014], du moins du phénomène mémoriel occidental, comme la célébration du dixième anniversaire du 11 Septembre 2001 a pu le montrer. Ces deux événements traumatiques ne sont pas comparables « en euxmêmes ${ }^{7}$; néanmoins, leurs modalités de commémorations se trouvent finalement très interconnectées en termes de déroulements. La commémoration du dixième anniversaire a coïncidé avec l'inauguration du mémorial du 11 Septembre, un espace paysager de trois hectares au sein duquel deux immenses bassins déversant de l'eau en permanence ont été érigés, comme véritables « points de repère » de l'endroit où se dressaient les tours du World Trade Center [Truc, 2015]. À l'instar des victimes de la Shoah, la plupart des défunts(es) n'ont pu être identifié(es) en raison de la violence inouïe du choc. Il s'agit donc, là aussi, de morts sans corps et sans sépulture. Les noms des 2983 personnes tuées lors des attentats ont été lus, un à un, ponctués par six moments de silence : à l'instant précis des attaques contre les tours et du Pentagone, à l'heure où l'avion s'est écrasé en Pennsylvanie et à l'heure où les tours se sont effondrées. Cette litanie des noms rappelle le rituel qui se déroule à Yad Vashem pour Yom Ha Shoah. La voix représente en effet une voie privilégiée pour figurer l'infigurable. Par ailleurs, la catégorisation des acteurs contemporains du 11 septembre emprunte symboliquement aux acteurs contemporains de la Shoah. On retrouve métaphoriquement les figures des témoins, des victimes et des bourreaux, ainsi que celle des « Justes ${ }^{8}$, qui ont sauvé des Américains au péril de leur vie. Cette dernière similarité

6. L'ossuaire de Douaumont, conçu au lendemain de l'armistice de 1918, abrite les restes de 130000 soldats inconnus.

7. Par ailleurs, la Shoah a été pensée pour rester invisible et cachée, tandis que les attentats du 11 Septembre ont été imaginés et conçus pour être mis en images et en films.

8. En 1953, la Knesset créa à la fois le mémorial de Yad Vashem à Jérusalem et le titre de Justes parmi les nations pour celles et ceux qui ont mis leur vie en danger pour sauver des Juifs. Le titre de Juste est décerné au nom de l'État d'Israël par le mémorial de Yad Vashem. Il s'agit d'une expression tirée du Talmud (traité Baba Batra, 15 b). 
catégorielle comprend les pompiers de New York et les passagers du vol 93 qui, grâce à leurs révoltes, ont empêché que l'avion ne tombe sur la Maison-Blanche. Les victimes du terrorisme, présentées comme des personnes ordinaires, succèdent, ou plutôt se surajoutent, aux États-Unis, aux victimes du nazisme en Europe. Par ces relations tissées en réseaux, le leitmotiv «May we never forget » est définitivement devenu un élément clé des discours politiques.

Les murs tapissés des visages des victimes, hommes, femmes et enfants, au National September 11 Memorial \& Museum rappellent le Mémorial des enfants juifs déportés de France du musée de la Shoah à Paris, ou La Tour des visages du musée de l'United States Holocaust Memorial Museum de Washington, tandis que les noms des victimes du 11 Septembre, gravés dans des plaques de bronze sur les margelles qui encadrent les deux bassins s'apparentent, par exemple, aux noms des victimes de la Shoah, gravés dans le marbre noir qui encercle le mémorial de l'Holocauste à Miami Beach.

Figures 1 et 2 - Noms gravés et National September 11

Memorial \& Holocaust Memorial Miami Beach
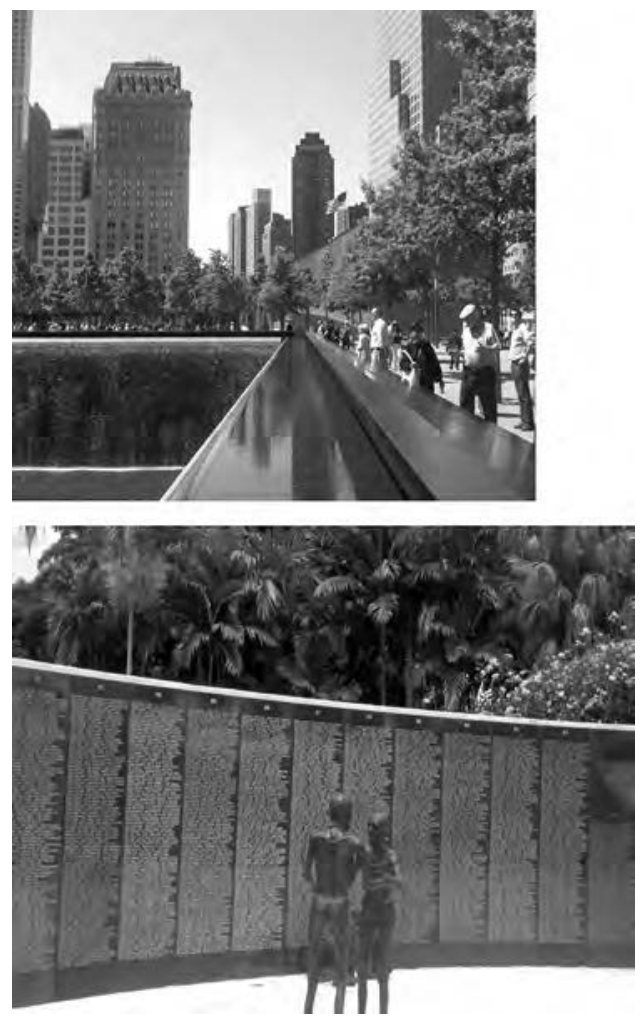

Source : (c Dominique Chevalier (mai 2012). 
Les familles des victimes elles-mêmes semblent revendiquer cette similitude avec la mémoire accordée aux victimes de l'Holocauste [Swartz, 2006, p. 95]. Et réciproquement, les événements du 11 Septembre ne sont pas sans effets sur la manière d'appréhender la mémoire de la Shoah. Ainsi, dans le sillage des attentats du 11 Septembre, Michaël Bernard-Donals a analysé les commentaires laissés par les visiteurs du grand musée nord-américain de la Shoah, l'United States Holocaust Memorial Museum de Washington. L'auteur montre combien les souvenirs s'amalgament et fait l'hypothèse que les événements contemporains agissent comme des écrans pour la compréhension des processus qui ont abouti à la Shoah [Bernard-Donals, 2005, p. 74]. De manière située à la fois spatialement et temporellement, l'Holocauste devient le 9/11, et inversement ; il parle, à ce sujet, de «mémoire oublieuse » (Forgetful Memory) et évoque, pour appuyer son propos, un commentaire anonyme laissé le 30 décembre 2001 dans lequel la personne dit comprendre, à l'aune des attentats du 11 Septembre, ce qu'être juif signifie.

À rebours de cette prégnance rituelle, mémorielle et muséographique, le National Museum of American Indian (NMAI) de Washington, a été pensé comme un lieu vivant dans lequel la mémoire autochtone s'exprime profondément à travers l'enseignement et la transmission des coutumes et des langues indiennes. La pierre angulaire du discours et de l'âme de l'endroit repose autant sur l'affirmation de la rémanence des Natives que sur le refus de céder à la victimisation ${ }^{9}$. Ce parti pris muséographique est d'ailleurs parfois critiqué par certains détracteurs qui lui reprochent de ne pas avoir été conçu comme un grand musée de l'Holocauste à la mémoire des Amérindiens [Mauze, Rostkowski, 2007].

\section{Rescapés, architectes et touristes : agents et, parfois, agitateurs mémoriels}

Dans le sillage de ce processus mémoriel se développe un tourisme compris comme pratique culturelle dans laquelle les touristes, avides de découvertes, prennent une part active dans le choix de leur destination [Équipe MIT, 2002]. Le tourisme de mémoire s'inscrit pleinement dans ce processus. Avec la disparition des derniers contemporains de la Shoah, peut-être allons-nous assister, dans les années à venir, à la fin de «l'ère du témoin » analysée par Annette Wieviorka [1998]. Raconter ce qui s'est passé et toucher les consciences afin de prévenir et de faire en sorte qu'aucun autre génocide ne puisse se (re)produire, telles sont bien les missions exprimées dans ces musées mémoriaux. Pour dire au plus près la barbarie et l'horreur, et pour prévenir leur retour, les architectes, les conservateurs(trices) misent sur l'originalité, la performativité des agencements et la

9. En septembre 2004, le National Museum of American Indian (NMAI), premier musée national exclusivement consacré aux Amérindiens, a été inauguré, au cœur de la capitale fédérale, non loin du Capitole. À la suite des commémorations liées à la «Découverte » de 1492, la tendance était de focaliser sur la vision des vaincus et la souffrance des peuples colonisés. Dans ce musée, seule la vision amérindienne est considérée comme authentique et prend valeur de vérité. L'accent est davantage mis sur le futur que sur le passé [Greenberg, 2007]. 
puissance narrative des dispositifs muséographiques généralement fondés sur l'expérience, lesquels mettent à rude épreuve les corps et les esprits des visiteurs. Par la pratique et l'apprentissage de ces lieux autres durant l'espace-temps de leur visite, ces derniers ont, à l'issue du parcours, bien souvent l'impression d'avoir « véritablement» côtoyé les lieux de l'inimaginable. Idéalement, à l'issue de l'expérience de la visite, chaque visiteur s'est métamorphosé en témoin, «un témoin délégué, un témoin de substitution, un vicarious witness » [Hartog, 2007, p. 13] susceptible de transmettre à son tour, au gré de ses mobilités et de son parcours individuels, les histoires et les mémoires exposées aux musées. À l'avènement de l'ère des témoins initiée par le procès Eichmann succéderaient ainsi des aires de production de témoins, pour peu que des starchitectes ${ }^{10}$ mondialisés en aient dessiné les édifices idoines en termes de matérialité, de performativité et d'agentivité, lesquels soutiennent par ailleurs un nouvel urbanisme métropolisé. Le starchitecte probablement le plus symptomatique du système mondialisé des stars-architectes et de leurs rôles dans la circulation des «modèles mémoriaux » et, d'une certaine manière, d'une « labellisation » de la Shoah comme expression de mémoires douloureuses issues d'un «passé chargé de laideur et de souffrances », comme il l'écrit lui-même dans son autobiographie, est probablement Daniel Libeskind [2005, p. 13]. À la suite du musée juif de Berlin, il dessine différents projets en lien avec le judaïsme et la Shoah, notamment le Danish Jewish Museum à Copenhague (2003), le Centre de la Shoah à Manchester et le Contempory Jewish Museum à San Francisco (2008). Il réalise également l'Imperial War Museum North dans l'agglomération de Manchester en 2002, et le musée d'histoire militaire de Dresde en 2009. Cette expérience en matière d'architecture mémorielle lui a valu d'être retenu pour la reconstruction du World Trade Center à New York.

Les musées, comme lieux de mémoire et de patrimoine, constituent des objets spatiaux à part entière. Ils combinent et impliquent en effet des stratégies discursives articulant des intentions, des émotions, des valeurs, des croyances et des appartenances plurielles, lesquelles agencent et produisent des identités individuelles ou collectives. Ces lieux mémoriels s'inscrivent dans des logiques spatiales multiples et complexes qui varient selon les niveaux scalaires auxquels elles sont appréhendées. Ces « hauts lieux », ou «lieux de condensation ${ }^{11}$ » au sens où l'entend Bernard Debarbieux [1995] sont produits, travaillés, tourmentés par des superpositions, des agencements de dynamiques morales, éducationnelles, diasporiques, politiques, géopolitiques, économiques et touristiques [Chevalier, 2014b].

10. Ce terme est un mot-valise utilisé pour décrire des architectes dont la célébrité et la critique les ont transformées en idoles du monde de l'architecture. Ce statut, outre qu'il leur donne une certaine notoriété auprès du grand public, est généralement associé à la nouveauté avant-gardiste.

11. Bernard Debarbieux en donne la définition suivante : « Les lieux de condensation, bien que motivés par les seules temporalités mythiques et symboliques, changent de nature et d'inscription. S'ils se maintiennent au cœur vibrant des nations d'autrefois, ils surgissent aussi, différents, là où de nouvelles nations s'affirment. [...] Mais dans la plupart des cas, leurs caractéristiques ont changé, bien davantage que leur référent. » 
Au travail et à l'influence des architectes et des divers entrepreneurs de mémoires, il convient également d'ajouter, comme dans le cas du Cambodge, le rôle des artistes. Sans la ténacité et la volonté de témoigner d'artistes tels que Rithy Panh, Vann Nath ou Séra notamment, et corrélativement sans les touristes et l'engouement pour le dark tourism [Lennon, Foley, 2010] en général et le tourisme génocidaire en particulier, les lieux de mémoire témoins du génocide ${ }^{12}$ perpétré par les Khmers rouges seraient sans doute restés à l'abandon.

Depuis la fin des années 1990, le tourisme, en croissance constante au Cambodge, s'organise autour de deux pôles extrêmes et antagonistes : Siem Reap où se situent les Temples d'Angkor classés au patrimoine mondial par l'Unesco depuis 1992, et Phnom Penh, la capitale, qui abrite notamment deux grands lieux de mémoire du génocide : Tuol Sleng, plus connu sous le nom de prison S21 et Chœung Ek. Cette coprésence antagoniste de splendeurs et de douleurs constitue les deux catégories structurantes de l'offre touristique. Il arrive qu'elles se superposent, tant la terre cambodgienne continue encore et toujours d'expulser des restes humains. En 2013, en effet, un nouveau charnier de plusieurs milliers de squelettes a été découvert dans la province de Siem Réap.

En 2012, Duch, le directeur de la prison S21 sous le régime de Pol Pot, « Duch, le Maître des forges de l'enfer » pour reprendre le titre du documentaire réalisé par Rithy Panh [2011], a été condamné, en appel, par la Cour suprême auprès des Chambres extraordinaires au sein des tribunaux cambodgiens (CETC), tribunal hybride parrainé par les Nations Unies, à la perpétuité. Sur les 16000 personnes détenues à Tuol Sleng, personne ne s'est échappé. À la libération du camp, le lieu ne comptait que sept survivants, parmi lesquels le peintre Vann Nath. Entre sa libération en 1979 et sa mort en 2011, ce dernier n'a cessé de peindre les scènes auxquelles il a assisté, à la fois pour témoigner, laisser des traces des horreurs perpétrées par les Khmers rouges dans ce génocide où les images de souffrances et de persécution restent rares, et pour que les jeunes Cambodgien(nes) apprennent et voient ce qu'aucun manuel scolaire n'expliquait et ne montrait jusque-là. Une vingtaine de toiles sont actuellement exposées au musée du génocide de S21.

Le génocide et la prison de Tuol Sleng sont aujourd'hui mondialement connus et entrés dans la mémoire collective grâce aux films de Rithy Panh, aux peintures de Vann Nath et au procès de Duch. Ils ne sont pas sans effet sur l'intérêt récent porté à ces tragiques lieux de mémoires et leur remise en état, qui relève d'une tendance hétérogène reposant à la fois sur un sentiment de malaise du point de vue cambodgien et sur un intérêt grandissant du point de vue Occidental.

12. La qualification de «génocide» pour évoquer les massacres commis sous le Kampuchéa démocratique n'a pas fait l'unanimité. Les termes de politicide, de crimes contre l'humanité ont parfois été utilisés pour évoquer les assassinats de masse commis par les Khmers rouges. Toutefois, après de nombreux aléas, le terme de génocide, omis volontairement dans les accords de Paris d'octobre 1991, a fait sa réapparition sur la scène internationale, en décembre 1997, lorsque l'ONU a reconnu qu'un quart de la population cambodgienne avait été victime d'un génocide entre 1975 et 1979. 
Depuis le 31 juillet 2009, les archives de la prison sont inscrites sur le Registre « Mémoires du Monde » de l'Unesco. C'est également le cas de Chœung Ek, situé à dix-sept kilomètres au sud-ouest de la capitale. Transférés de nuit par camions, les prisonniers détenus à Tuol Sleng y étaient amenés, exécutés et enterrés dans des fosses communes. Les nourrissons et enfants en bas âge avaient la tête fracassée contre les arbres. L'endroit, couramment appelé Killing Fields par les touristes, est géré par la société privée JC Royale Co., qui a obtenu un bail jusqu'en 2035 pour l'exploitation du site.

Ces lieux associent des témoignages, des expositions d'objets, des expositions permanentes, des programmes éducatifs, des cérémonies commémoratives, et l'édification d'architectures ou compositions symboliques : stupa empli de crânes et Maison des esprits à Chœung Ek, carte du Cambodge réalisée à partir de crânes, par Vann Nath et trois rescapés de S21, à Tuol Sleng, pour figurer « le Kampuchea démocratique de Pol Pot comme un immense charnier » [Burnet, 2013] ; le Mékong et le Tonlé Sap y sont peints en rouge pour évoquer les nombreux corps charriés par ces fleuves. Le curseur discursif pour évoquer l'horreur de ce qui s'est déroulé en ces lieux est délicat puisqu'il convient à la fois de rester suffisamment pédagogique pour le public composé de scolaires et de touristes de plus en plus intéressés par un tourisme de mémoire international (mais souvent étrangers aux cultures locales), sans toutefois diluer la dimension commémorative et symbolique que les survivant(es) ou personnes ayant des liens personnels avec l'ampleur des crimes commis viennent précisément y rechercher, et sans par ailleurs trop compromettre les discussions sur la paix et la réconciliation.

Si, après de longues années de quasi-délaissement, le DC-Cam (Documentation Center of Cambodia), aidé financièrement par le Congrès des États-Unis, a largement contribué à redynamiser le musée du Génocide de Tuol Sleng [Margolin, 2007], les relations tissées en réseaux coopératifs semblent également se tourner vers l'Asie. En effet, en 2012, la prison S21 a organisé, en partenariat avec le Musée Mémorial de la Paix de la préfecture d'Okinawa du Japon, une exposition autour de la thématique de la paix. Sa présidente, Reiko Goya, a affirmé à cette occasion que l'exposition symbolisait un message important adressé aux peuples cambodgien et japonais, leur rappelant le souvenir de la guerre et les appelant à lutter ensemble pour prévenir la guerre. De son côté, Hiroshi Kowamura, ambassadeur du Japon au Cambodge, a souligné combien l'exposition avait nécessité un travail de longue haleine, le partenariat entre les deux musées s'étant étalé sur trois ans.

Ces sites de massacres, aujourd'hui transformés en musées ouverts, constituent des lieux d'apprentissage de l'histoire du génocide cambodgien, des lieux de recueillement et des lieux de mémoire, nationaux et internationaux. Mais, dans ce pays qui commémore très discrètement le souvenir de la prise de Phnom Penh par les Khmers rouges, étant donné que certains politiques actuellement au pouvoir 
Figure 3 - Carte du Cambodge réalisée à partir de crânes - Tuol Sleng

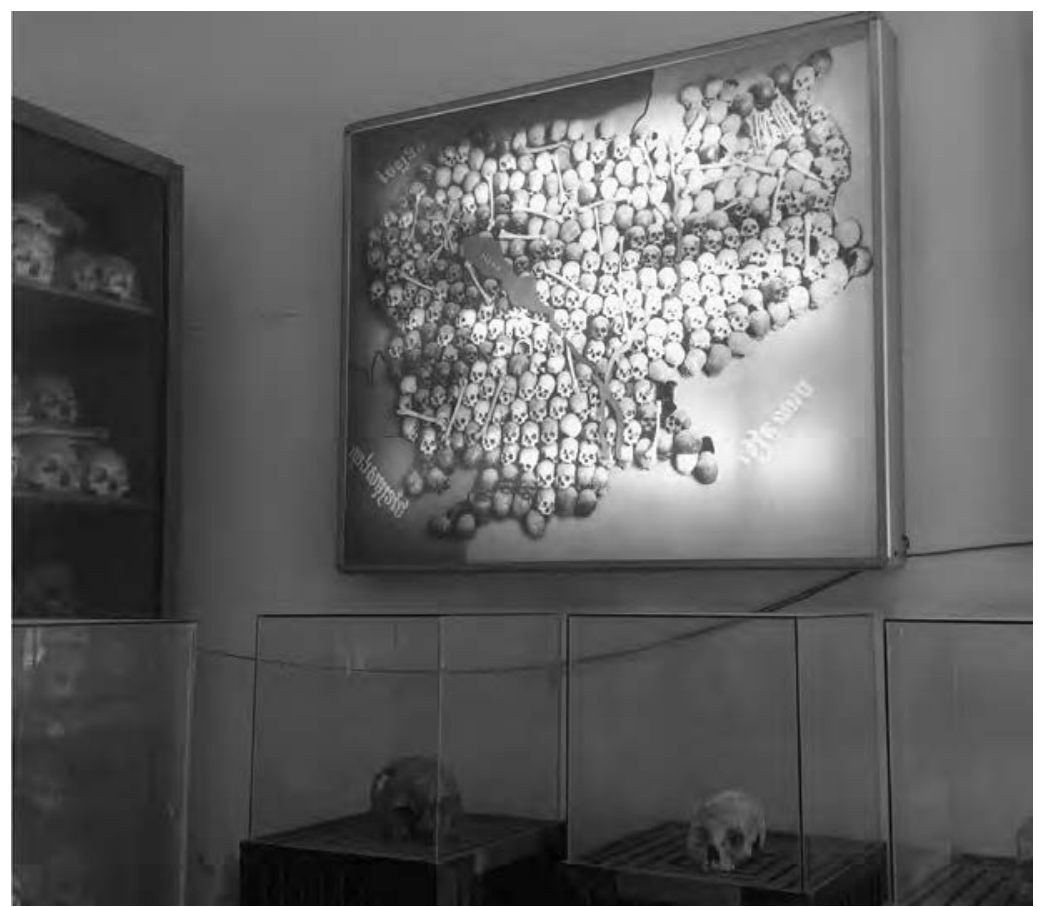

Source : ( ) Dominique Chevalier (février 2014).

sont d'anciens Khmers rouges ${ }^{13}$, de nouvelles formes de commémoration cherchent à s'exprimer. À l'occasion du quarantième anniversaire de la chute de la capitale, le premier mémorial autonome « hors site », appelé « À ceux qui ne sont plus là » par l'artiste, aurait dû être inauguré le 17 avril 2015, sur la place située en face de l'Ambassade de France, à Phnom Penh. Cette œuvre, conçue et voulue par l'artiste franco cambodgien Séra comme un « lieu de mémoire et de recueillement pour tous ceux qui souhaitent honorer leurs disparus ${ }^{14}$ », reconnue par ailleurs par les CETC (Chambres extraordinaires au sein des tribunaux Cambodgiens) comme une réparation officielle pour les victimes des Khmers rouges, répond à la fois à l'attente des parties civiles du procès des dirigeants Khmers rouges et à celles de nombreux groupes internationaux œuvrant pour la justice et la reconnaissance de ce génocide. En avril 1975, lorsque les Khmers rouges entrent dans la capitale, Séra, âgé de treize ans, et sa mère se réfugient à l'Ambassade de France et parviennent ensuite à fuir le Cambodge. Ce ne sera malheureusement pas le cas de son père, de nationalité cambodgienne. Dans un entretien [Séra, 2014], l'artiste explique que c'est précisément pour lui et les autres disparus qu'il travaille sans relâche depuis trente ans.

13. C'est notamment le cas du Premier ministre Hun Sen qui critique la culture du souvenir du drame au nom de l'unité nationale.

14. Association Anou'savry Thom, Communiqué de presse du 3 juin 2014. 
Malgré l'opportunité de faire coïncider l'inauguration de ce nouveau mémorial avec la date anniversaire du quarantenaire, cette cérémonie commémorative n'a cependant pas eu lieu, en raison d'un conflit qui oppose l'artiste et la mairie de Phnom Penh. Dans cet affrontement, la façon dont les divers acteurs tissent des liens symboliques réels ou idéels avec les lieux et avec les pratiques patrimoniales mémorielles joue un rôle crucial. En effet, de son côté, la mairie souhaite soustraire la présence du mémorial au regard du plus grand nombre, notamment des citadins et des passants, et par conséquent, désire le cantonner dans l'espace mémoriel déjà existant de Chœung Ek, au prétexte que l'endroit est déjà fortement «mémorialisé », patrimonialisé et donc aménagé pour les pratiques touristiques. Les fosses, dont les limites ne sont pas toutes stabilisées, sont en partie recouvertes, et des panneaux demandent aux visiteurs de ne pas toucher aux restes humains pouvant affleurer à la surface du sol. Des photographies, exposées à proximité des fosses, et l'affichage du décompte des corps et squelettes dégagés témoignent, sur la plupart des fosses communes, de l'ampleur des crimes. C'est donc en ce haut lieu mémoriel, excentré de la capitale, que la mairie de Phnom Penh souhaiterait ancrer le mémorial « À ceux qui ne sont plus là ». En revanche, Séra considère cette décision d'implanter son mémorial à cet endroit-là comme un double déni de l'histoire ; d'une part parce que cela l'enclaverait en périphérie de la capitale, le confinerait dans un lieu aujourd'hui spécifiquement mémoriel, le soustrayant aux regards quotidiens des Cambodgiens et les disculpant des responsabilités politiques, et d'autre part parce qu'une partie des victimes de Chœung Ek étaient des Khmers rouges, accusés par d'autres Khmers rouges de trahir les idéaux du Kampuchea démocratique. Il souhaiterait donc, au regard de tous ces arguments, ancrer le mémorial au cœur de l'espace public de la capitale, entre le lycée Descartes et la Bibliothèque nationale.

Figure 4 - Chœung Ek. Charnier recouvert et photographies comme témoignages

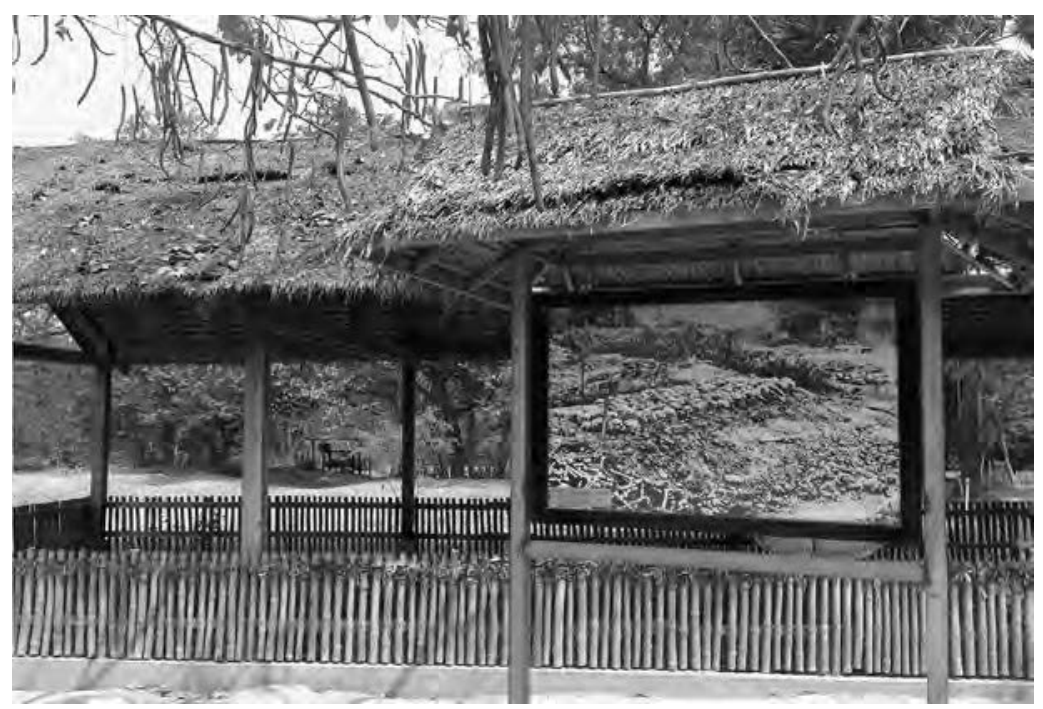

Source : (C) Dominique Chevalier (février 2014). 
Figures 5 et 6 - Localisation souhaitée par Séra

Source : Google Maps.

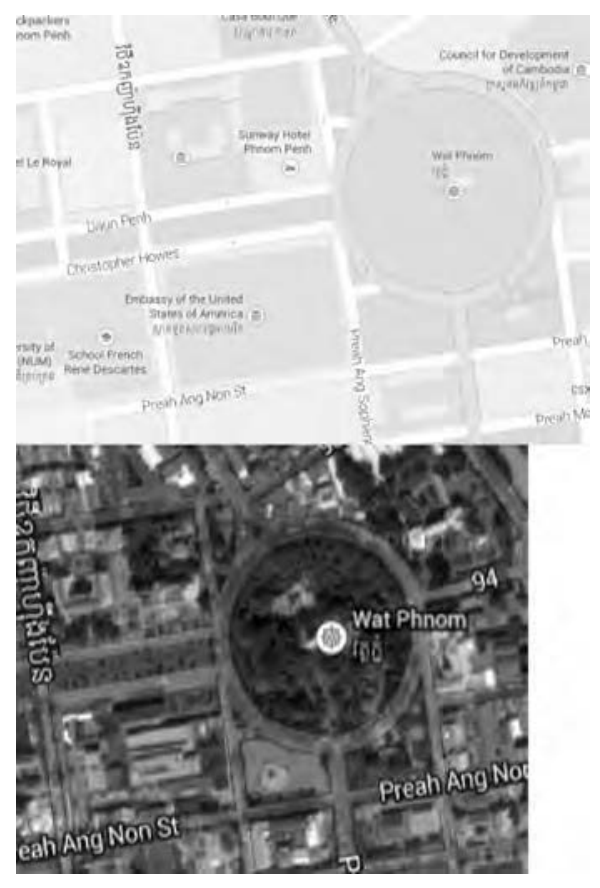

Le site de Chœung Ek répond à une volonté de commémoration officielle qui s'insère dans une géopolitique à plusieurs niveaux scalaires, et dans un contexte où le tourisme se développe rapidement. Entre 2011 et 2012, celui-ci a augmenté de près de $25 \%$ (analyses du ministère du Tourisme cambodgien). Tout comme pour Angkor, la politique du gouvernement est clairement d'insérer ce lieu de mémoires douloureuses dans une dynamique touristique. Dans les années 1980, le site était resté à l'écart de toute commémoration, à la fois en raison de son relatif éloignement de la capitale dans un contexte de pénurie de transports, mais également parce que le caractère macabre du site rappelait aux vivants la réalité du génocide et évoquait, selon la culture cambodgienne, l'errance des âmes des disparus(es) n'ayant pas encore trouvé le repos. La signature des Accords de Paris sur le Cambodge du 23 octobre 1991 mettant fin à la guerre civile entre les forces de l'État du Cambodge d'une part, et les Khmers rouges et autres factions d'autre part, marque le début d'une scénographie du lieu. La plupart des ossements ont été rassemblés dans un stupa, par couches superposées sur 17 niveaux en fonction de l'âge et du sexe. Ces crânes séparés, dont certains portent encore un reste de bandeau sur les trous oculaires, constituent avec les quelques photos prises par les Vietnamiens lors de leur arrivée à $\mathrm{S}-21$, l'essentiel des images du génocide. 
Figure 7 - Base inférieure du stupa de Chœung E

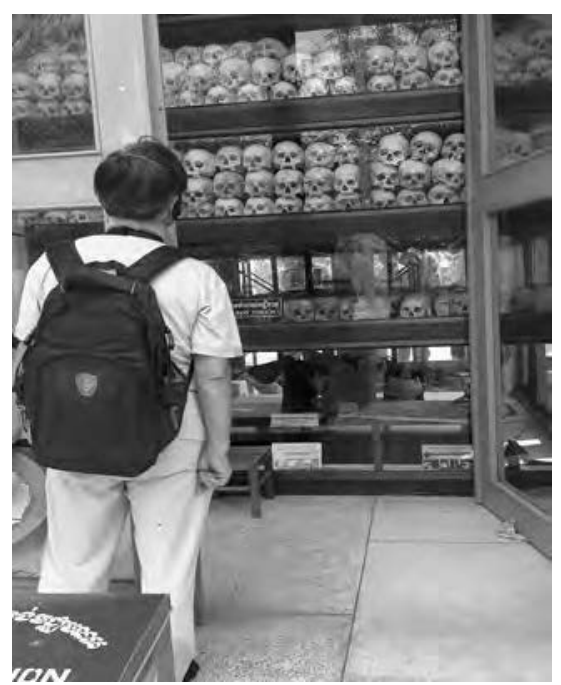

Source : ๑ Dominique Chevalier (février 2014).

Au-delà des enjeux mémoriels et patrimoniaux, le conflit lié à l'emplacement du mémorial de Séra masque en réalité des enjeux d'ordre politique.

\section{Massification touristique et marchandisation : deux réalités inhérentes à la construction des patrimoines ?}

À la définition classique de patrimoine, généralement associée à la notion d'héritage, se greffe une acception politisée qui s'intéresse au patrimoine en tant qu'objet coproduit par différents acteurs en fonction d'objectifs plus ou moins explicites. Le développement des politiques en matière de tourisme culturel s'ancre dans une volonté multiforme d'allier développement économique, visites du patrimoine, pratiques culturelles, marché de biens et services, échanges culturels et transmission d'une mémoire, lesquels coproduisent de nouveaux patrimoines. Le tourisme mémoriel s'appuie sur des discours nationaux, régionaux ou communautaires multivoques, économiques, patrimoniaux, et culturels qui mettent en exergue ces demandes. Les sites liés aux mémoires douloureuses agrègent à ces divers enjeux des dimensions singulières qui remettent en question sans fin les principes de valeurs universelles. La sempiternelle question des effets et des risques de dévoiement liés à la mise en tourisme [Burnet, 2013] s'impose avec force lorsqu'elle concerne ces lieux de mémoire spécifiques [Chevalier, Lefort, 2016].

Sur un autre terrain des mémoires douloureuses, les tunnels de $\mathrm{Cu} \mathrm{Chi}$, au Vietnam, réunissent différents aspects qui rendent le lieu particulièrement intéressant pour les questionnements qui nous préoccupent. En effet, ils représentent (en creux) le martyre du peuple vietnamien, mais surtout ils incarnent l'ingéniosité 
de sa résistance ${ }^{15}$. Situé à une cinquantaine de kilomètres au nord du centre d'Hô Chi Minh-Ville (ex. Saigon), le complexe militaire de Ben Dinh, devenu un site touristique très connu, au programme de nombreux tours organisés, montre une partie de ces tunnels, «symbole [s] de la guerre contre les Américains » [Giblin, 2007, p. 13]. Ces tunnels dissimulaient, sur plus de deux cents kilomètres des cachettes de 60 à 70 centimètres de large, sur une hauteur de 80 à 90 centimètres. Des trappes, camouflées avec soin, en masquaient les accès, de faux sols pivotants aboutissant sur des rangées de bambous finement acérés servaient à piéger l'ennemi. Ces réseaux de tunnels, bien connectés à l'arrivée de la piste Hô Chi Minh, rejoignaient à l'extérieur, des postes de tir. À l'intérieur, de longues galeries servaient de cuisines, de dortoirs, de salles de réunion et de poste d'opérations chirurgicales. À un niveau inférieur, des salles plus petites constituaient des abris en cas de bombardements. Nourriture, armes et munitions y étaient entreposées. Véritable petite ville abritant 16000 personnes au plus fort de la guerre, ces réseaux souterrains ont joué un rôle crucial dans la résistance et la victoire vietnamienne.

Depuis quelques années, le Vietnam est devenu un haut lieu du tourisme mondial, et Hô Chi Minh-Ville s'affirme comme un pôle touristique, économique et culturel majeur. Le tourisme représentait $11 \%$ du PIB municipal en 2013, et en 2014, les chiffres des cinq premiers mois de l'année ont montré une hausse de $9,7 \%$ en comparaison avec la même période de l'année précédente ${ }^{16}$. Comme dans la plupart des lieux mémoriels, le site cumule plusieurs fonctions : transmettre (un peu) le souvenir de la guerre, de ses douleurs, de ses combats, et conforter l'identité d'un peuple combattant, ingénieux et victorieux.

Le complexe militaire de Ben Dinh est désormais aménagé pour les touristes, et pour 80000 dongs (environ 3,50 euros) les visiteurs peuvent accéder au site qui s'apparente davantage à un «parc à thème » qu'à un mémorial. Le parcours commence par le visionnage d'un film qui permet de situer le contexte politique et géographique du lieu à l'époque de la guerre, et de percevoir aujourd'hui la dimension idéologique de l'ensemble. Le reste du parcours se fait accompagné d'un guide, habillé en treillis kaki couleur de la jungle et chaussé des célèbres sandales noires en caoutchouc, laissant des empreintes «neutres » dans le sol. Différentes scènes, peuplées de quelques mannequins vêtus de treillis kaki ou noirs, mettent en valeur l'héroïsme du Viet Cong et le quotidien des soldats. Les guides montrent l'ingéniosité mise en œuvre dans la construction de ces tunnels (pièges, trappes, fumées de cuisine camouflées et évacuées beaucoup plus loin...) et n'hésitent pas à se mettre eux-mêmes en scène pour évoquer l'agilité des Vietnamiens.

15. Pour trouver trace des horreurs de la guerre, c'est au musée des vestiges de la guerre qu'il faut se rendre, où le premier étage est notamment consacré aux effets et aux conséquences de l'utilisation de l'agent orange sur la population et sur les espaces, et aux agressions et atrocités commises par les Américains. Notons par ailleurs que la question des Boat People n'est jamais abordée.

16. Consulat général de France, Synthèse des articles de presse. Semaine de 26 au 30 mai 2014. 
Figure 8 - Guide montrant l'entrée dans un tunnel

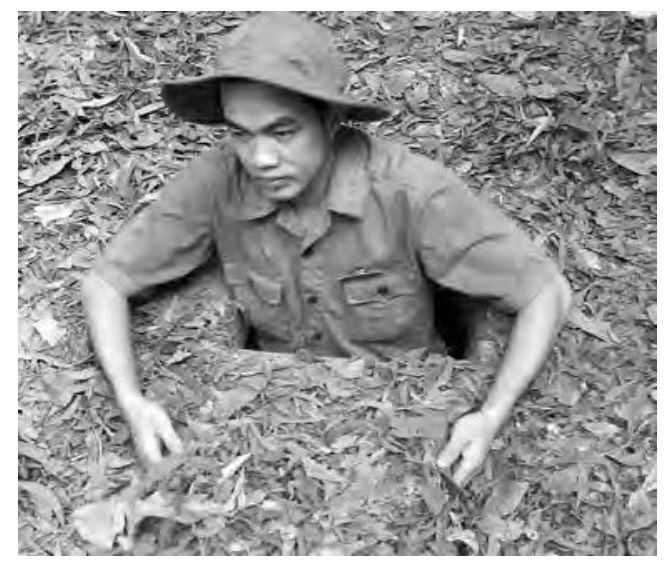

Source : ๑ Dominique Chevalier (janvier 2013).

Une portion de tunnel, ouverte sur une section de cent mètres aux touristes volontaires et non claustrophobes, a été élargie pour épouser les corpulences occidentales. Tous les vingt mètres, une sortie permet de s'en extraire. L'expérience est éprouvante pour beaucoup de volontaires qui se découvrent claustrophobes et

Figures 9 et 10 - Transformation de l'échoppe entre 2013 et 2015

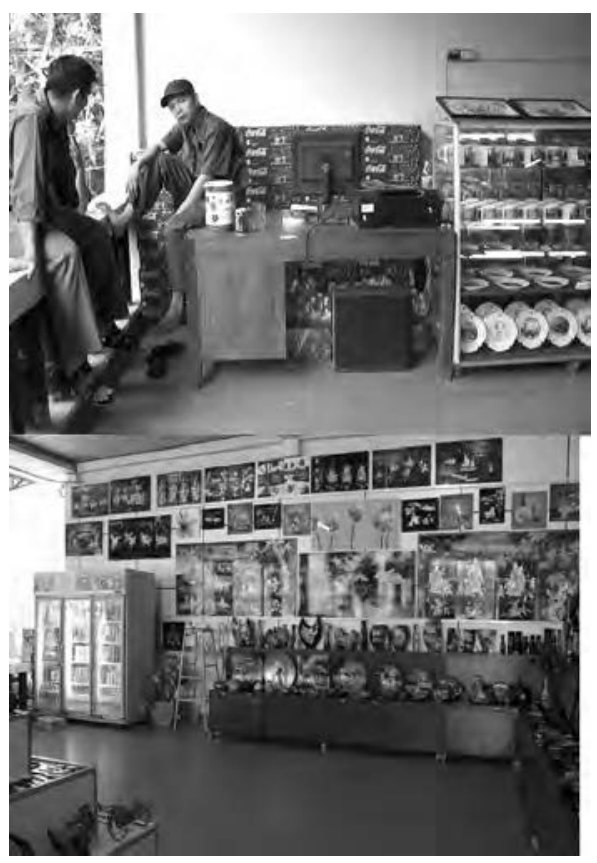

Source : ๑ Dominique Chevalier (janvier 2013 et avril 2015). 
paniquent à l'idée de ne pas avoir la place de faire marche arrière pour revenir sur leurs pas. La transformation de l'échoppe où sont vendus divers souvenirs aux touristes entre 2013 et 2015 témoigne de la montée en puissance de la fréquentation des lieux. À la petite échoppe proposant Coca Cola, sandwichs et quelques menus souvenirs exposés derrière une vitrine a succédé une boutique plus grande, richement aménagée, beaucoup mieux achalandée en souvenirs diversifiés (objets, artisanat...) et accessibles à portée de main. À proximité se trouve un stand de tir où les moins pacifistes peuvent s'essayer au tir avec d'authentiques armes de guerre de l'époque. Les intéressés ont le choix entre six armes : kalachnikovs (AK), fusil-mitrailleur américain (M16), carabine, mitrailleuse lourde M60, AR15 et M30. Il faut acheter dix balles au minimum.

À l'issue de la visite de ce qui fut l'un des sites les plus impitoyables et les plus sanglants de la guerre du Vietnam, les visiteurs auront, avec plus ou moins de conviction «joué à la guerre » sans toutefois être vraiment perturbés par cette expérience ; l'émotion tient peu de place dans ce parc où les horreurs du passé semblent frappées d'amnésie. Depuis plusieurs années, le Vietnam s'invente pour devenir un haut lieu du tourisme mondial. L'artiste Dinh Q. Lê voit dans cette propagande touristique sourde et muette aux batailles les plus acharnées un « conflit de messages » [Choron-Baix, 2009]. En 2016, le nombre de touristes a dépassé les dix millions; c'est pratiquement dix fois plus qu'il y a dix ans.

Figure 11 - Classement TripAdvisor des sites évoqués

\begin{tabular}{|c|c|c|}
\hline LIEUX ÉVOQUÉS DANS CET ARTICLE & $\begin{array}{l}\text { CLASSEMENT } \\
\text { CHOSES À VOIR }\end{array}$ & $\begin{array}{l}\text { NOMBRE } \\
\text { D'AVIS }\end{array}$ \\
\hline $\begin{array}{l}\text { Musée de Tuol Sleng, Phnom Penh } \\
\text { (Cambodge) }\end{array}$ & 4 sur 131 & 3908 \\
\hline Chœung Ek, Phnom Penh (Cambodge) & 1 sur 131 & 5076 \\
\hline Tunnels de Cu Chi (Vietnam) & 2 sur 191 & 3911 \\
\hline Holocaust Memorial Miami Beach (États-Unis) & 6 sur 149 & 1034 \\
\hline Memorial 9/11, New York (États-Unis) & $6 \operatorname{sur} 1622$ & 17663 \\
\hline USHMM, Washington (États-Unis) & 5 sur 463 & 3815 \\
\hline Mémorial de la Shoah, Paris (France) & 41 sur 1119 & 374 \\
\hline Mémorial de Yad Vashem, Jérusalem (Israël) & $2 \operatorname{sur} 232$ & 3163 \\
\hline Musée des Réfugiés juifs, Shanghai (Chine) & 19 sur 1301 & 219 \\
\hline Centre du Mémorial, Kigali (Rwanda) & 1 sur 39 & 653 \\
\hline $\begin{array}{l}\text { Musée national Auschwitz - Birkenau } \\
\text { (Pologne) }\end{array}$ & 1 sur 3 & 3279 \\
\hline
\end{tabular}

Source : TripAdvisor 
Les musées, sites et mémoriaux consacrés aux mémoires douloureuses évoqués dans cet article sont généralement très bien classés sur le site Tripadvisor qui s'autoproclame «plus gros site de voyage sur le Web». Un aperçu, à la date du 28 mars 2015 permet de s'en rendre compte. Ce classement, bien qu'il puisse paraître arbitraire, n'est pas sans conséquence. En effet, les travaux réalisés par J. Miguéns, R. Baggio et C. Costa [2008] ont montré, à partir du cas de la ville de Lisbonne, l'importance des commentaires en ligne et l'impact du réseautage sur l'industrie du voyage. En 2009, l'Unesco s'est d'ailleurs associé à Trip Advisor, afin de valoriser les sites du patrimoine mondial auprès des 25 millions d'internautes navigant mensuellement dans les pages du site de voyage. L'objectif de ce partenariat consiste également à permettre aux gestionnaires de ces sites d'obtenir une évaluation de la part des visiteurs [Marcotte, Bourdeau, 2010].

\section{Conclusion}

Que retenir de cette exploration de quelques lieux de mémoires, patrimonialisés sous l'effet de jeux d'acteurs multiples, qui portent le traumatisme et la volonté de témoigner en héritage ? Véritables construits sociaux, ces musées et mémoriaux conservent, préservent, utilisent et diffusent, pour des activités pédagogiques, scientifiques et touristiques, des collections de témoignages, d'objets, d'archives uniques d'un point de vue historique. Divers objectifs accompagnent ces mises en patrimoine : « refroidir » la mémoire lorsque celle-ci est encore (trop) vive, parfois occulter certains faits, réparer - un peu - les blessures, témoigner, et, constamment, prévenir afin que de telles souffrances ne se reproduisent plus. Les projets consistent à graver des noms dans le marbre noir, la pierre de Jérusalem ou les plaques de bronze, à exhumer les restes et immortaliser ainsi celles et ceux qui sont mort(es) sans laisser de traces. À la fois racines et rhizomes, leurs souvenirs s'enracinent dans ces lieux qui leur sont spécialement dédiés, affleurent à la surface et tracent, plus ou moins vite et plus ou moins loin, les contours de notre humanité. Ces lieux de mémoires douloureuses appartiennent incontestablement au patrimoine de l'humanité, et comme l'a noté Sophie Wahnich [2011], il s'agit d'une «patrimonialisation du négatif qui habite les sociétés ». Non seulement ces musées exposent et transmettent des objets, des témoignages et des archives du passé pour préserver l'authenticité des diverses mémoires douloureuses et démontrer la véracité des processus génocidaires, mais ils créent aussi de manière dynamique un héritage plus ou moins commun autour de questionnements relevant des rapports passé/présent/futur en patrimonialisant «ce qui est voué au refoulement » [Wahnich, 2011]. Ils espèrent et ils suscitent des désirs de «plus jamais ça », répétés à l'envi et en toutes langues sur les livres d'or des différents musées consacrés aux mémoires traumatiques. Ce leitmotiv fait office de conjuration incantatoire pour les jeunes générations présentes et à venir. La mort de masse et la masse des morts qualifient de ce point de vue les lieux qui les abritent et les liens qui les rattachent aux vivants. La circulation et l'enracinement des cendres, des terreaux, des reliques ou des cadavres, la monstration des 
objets, la diffusion des témoignages et la fréquentation touristique participent, à différentes échelles, de la visibilité politique et de la vie sociale de ces lieux soutenus par la mémoire et la commémoration. La transmission des conditions ayant permis la mise en place de morts de masse et la circulation du patrimoine mémoriel qui lui est associé accompagne la fabrique d'un corps politique. Les individus, les touristes, les visiteurs éprouvent et contribuent à cette circulation idéelle et mémorielle mondiale. Tels des rhizomes, ils diffusent et alimentent les processus de territorialisation, déterritorialisation et reterritorialisation des différentes mémoires comme matériaux et substances du patrimoine collectif.

\section{Bibliographie}

Ashworth G. [2012], « The heritage as the built environment as development paradigms, possibilities and problems » in Lawrence R.J., TuRgut H., Kellett P., Requalifying the built environment : challenges and responses, Boston (Mass.), Hogrefe Publishing.

BERLINER D. [2005], « The abuses of memory : reflections on the memory boom in anthropology », Anthropoligical Quarterly, vol. 78, nº 1, p. 197-211.

Bernard-Donals M. [2005], "Conflations of memory or, what they saw at the Holocaust Museum after 9/11», The New Centennial Review, vol. 5, n 2, p. 73-106.

Burnet J. [2013], «Cambodge : l'ambiguïté politique et touristique des lieux de mémoire », Institut des Hautes Études sur la Justice : www.ihej.org/cambodge-lambiguite-politique-ettouristique-des-lieux-de-memoire/3/ (page consultée le 28 mars 2015).

Chevalier D., Lefort I. [2016], «Le touriste, l'émotion et la mémoire douloureuse », in Guinard P., TratnjeK B., Géographies, géographes et émotions, Carnets de géographes, n 9 : http://cdg.revues.org/644 (page consultée le 28 mars 2015).

Chevalier D. [2014a], «Édification de musées et mémoriaux consacrés à la Shoah : enjeux territoriaux, mémoriels, idéologiques et identitaires. Les exemples de Jérusalem, Paris, Berlin et Washington », in FOURNIER M. (dir.), Labellisation et mise en marque des territoires, Clermont-Ferrand, Presses universitaires Blaise Pascal, p. 561-580.

Chevalier D. [2014b], «Les musées urbains de la Shoah comme objets d'enjeux géopolitiques et espaces-temps de l'entre-deux », Revue EspaceTemps.net. : www.espacestemps.net/articles/les-musees-urbains-de-la-shoah-comme-objets-denjeux-geopolitiques-et-espace-tempsde-lentre-deux/ (page consultée le 28 mars 2015).

Choron-Baix C. [2009], «Le vrai voyage. L'art de Dinh Q. Lê entre exil et retour », Revue européenne des migrations internationales, vol. $25, \mathrm{n}^{\mathrm{o}} 2$, p. 51-68: http://remi.revues.org/ 4948 (page consultée le 15 janvier 2017).

Chretien J-P, Vidal C. [2014], «Un historien face au génocide des Tutsi », Vingtième siècle, vol. 2, n ${ }^{\circ} 122$, p. 23-35.

Cyrulnik B. [2001], Les Vilains Petits Canards, Paris, Odile Jacob.

Cyrulnik B., SERON C (dir.) [2004], La Résilience ou comment renaître de sa souffrance, Paris, Fabert.

Debarbieux B. [1995], «Le lieu, le territoire et trois figures de rhétorique », Espace géographique, vol. 24, n ${ }^{\circ}$ 2, p. 97-112.

Dumas H. [2014], Le Génocide au village. Le massacre des Tutsi au Rwanda, Paris, Seuil. 
ÉQuiPe Mobilités, ItinÉRAIRES, TerritoIREs (MIT) [2002], Tourismes 1. Lieux communs, Paris, Belin.

Gensburger S. [2010], Les Justes de France. Politiques publiques de la mémoire, Paris, Presses de SciencesPo.

GibLIN B. [2007], « Le tourisme : un théâtre géopolitique ?», Hérodote, vol. 4, n 127, p. 3-14.

GREENBERG R. [2007], «La représentation muséale des génocides », Gradhiva, n ${ }^{\circ} 5$ : https://gradhiva.revues.org/758 (page consultée le 15 janvier 2017).

Hall E.T. [1978], La Dimension Cachée, Paris, Points.

Hartog F. [2007], Évidence de l'histoire. Ce que voient les historiens, Paris, Gallimard.

Hilberg R. [1988] La Destruction des Juifs d'Europe, Paris, Arthème Fayard.

KAlisky A. [2004], «Mémoires croisées : références à la Shoah dans le travail de deuil et de mémoire du génocide des Tutsis », Revue Humanitaire (Médecins du monde), $\mathrm{n}^{\circ} 10$, numéro spécial à l'occasion de la commémoration des dix ans du génocide des Tutsi du Rwanda, p. 69-92 : http://aircrigeweb.free.fr/ressources/rwanda/Rwanda_A.Kalisky_human.html (page consultée le 15 janvier 2017).

KLARSFELD S. [2002], préface à Marianne Rubinstein, Tout le monde n'a pas la chance d'être orphelin, Paris, Gallimard.

Lalieu O. [2001], «L'invention du "devoir de mémoire" », Vingtième siècle, n o 69, p. 83-94.

LAPIERRE N. [2007], «Le cadre référentiel de la Shoah», Ethnologie française, n ${ }^{\circ} 37$, p. $475-482$.

LAVABRE M.-C. [2014], « La commémoration : mémoire de la mémoire ? », Bulletin des bibliothèques de France (BBF), n ${ }^{\circ}$ 3, p. 26-37: http://bbf.enssib.fr/consulter/bbf-2014-030026-002 (page consultée le 15 janvier 2017).

LAZZAROTTI O. [2010], «Tourisme culturel et patrimoine : quelques analyses pour un Monde habitable », Articulo - Journal of Urban Research: http://articulo.revues.org/1509 (page consultée le 5 décembre 2016).

Ledoux S. [2012], Écrire une histoire du « devoir de mémoire », Le Débat, n 170, p. 175-185.

LENNON J., Foley M. [2010], Dark tourism. The attraction of death and disaster, Boston (Mass.), Cengage Learning.

LibESKInd D. [2005], Construire le futur d'une enfance polonaise à la Freedom Tower, Paris, Albin Michel.

Lindenberg D., Garapon A., Padis M.-O. [2008], «Les ondes de choc de la Shoah », Esprit, $\mathrm{n}^{\circ} 3$, p. $80-87$.

Lowenthal D. [1985], The past is a foreign country, Cambridge, Cambridge University Press.

Marcotte P., Bourdeau L. [2010], «La promotion des sites du Patrimoine mondial de l'Unesco: Compatible avec le développement durable? », Management \& Avenir, $\mathrm{n}^{\circ} 34$, p. $270-288$.

MARGOLIN J.-L. [2007], «L'histoire brouillée. Musées et mémoriaux du génocide cambodgien », Gradhiva, n 5 , p. 84-95 : http://gradhiva.revues.org/781 (page consultée le 15 janvier 2017).

Mauze M., Rostkowski J. [2007], «La fin des musées d'ethnographie ? Peuples autochtones et nouvelles perspectives muséales », Le Débat, nº 147, p. 80-90.

Miguens J., Baggio R., Costa C. [2008], Social media and tourism destinations : Tripadvisor casestudy : www.iby.it/turismo/papers/baggio-aveiro2.pdf (page consultée le 28 mars 2015). 
NJanjo C. [2016], « À Kigali, Netanyahu évoque le "lien historique" du génocide », Le Point Afrique : http://afrique.lepoint.fr/actualites/a-kigali-netanyahu-evoque-le-lien-historique-dugenocide-07-07-2016-2052700_2365.php (page consultée le 15 janvier 2017).

PanH R. [2011], Duch, le maître des forges de l'enfer, documentaire, Paris, Éditions Montparnasse.

Perego S. [2010], «Les commémorations de la destruction des Juifs d'Europe au Mémorial du martyr juif inconnu du milieu des années cinquante à la fin des années soixante », Revue d'histoire de la Shoah, $\mathrm{n}^{\circ}$ 193, p. 471-507.

Perez M. [2016], « Comment Benyamin Netanyahou a pris l'Afrique par les sentiments », Jeune Afrique, 7 juillet 2016.

Petonnet C. [1982], «L'observation flottante. L'exemple d'un cimetière parisien », vol. 22, $\mathrm{n}^{\mathrm{o}}$ 4, Études d'anthropologie urbaine, p. 37-47 : www.persee.fr/doc/hom_0439-4216_1982_ num_22_4_368323 (page consultée le 15 janvier 2017).

Pollak M. [1993], Une Identité blessée. Études de sociologie et d'histoire, Paris, Métailié, p. 15-39.

Ricoeur P. [2000], La mémoire, l'histoire, l'oubli, Paris, Seuil.

Rousso H. [2015], «Un voyage au Rwanda», À la recherche du temps présent: http://tempresent.hypotheses.org/ (page consultée le 26 mars 2015).

SwARTZ A.K. [2006], « American art after September $11:$ a consideration of the Twin Towers », symploke, vol. 14, $\mathrm{n}^{\circ} 1-2$, p. 81-97.

Todorov T. [1995], Les Abus de la mémoire, Paris, Arléa.

TRUC G. [2015], «Venir à Ground Zero, se souvenir du 11 Septembre », EspacesTemps.net : www.espacestemps.net/articles/venir-a-ground-zero-se-souvenir-du-11-septembre/ (page consultée le 15 janvier 2017).

VIDAL C. [2014], «Enquêtes au Rwanda. Questions de recherche sur le génocide tutsi », Agone, $\mathrm{n}^{\mathrm{o}} 53$, p. 103-142.

WAHNICH S. [2011], «L'impossible patrimoine négatif », Les cahiers Irice, n ${ }^{\circ}$ 7, p. 47-62: https://www.cairn.info/revue-les-cahiers-irice-2011-1-page-47.htm (page consultée le 5 décembre 2016).

Wahnich S. [2007], «Transmettre l'effroi, penser la terreur. Les musées d'une Europe déchirée », Gradhiva, n ${ }^{\circ}$ 5, p. 26-37.

Wieviorka A. [1998], L'ère du témoin, Paris, Plon.

YeruShalmi Y.H. [1984], Zakhor. Histoire juive et mémoire juive, traduction française Éric Vigne, Paris, Gallimard. 